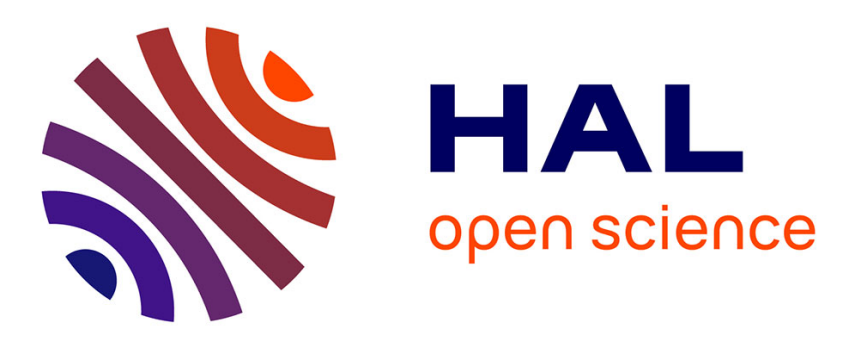

\title{
Split-step wavelet with local operators for the 3D long-range propagation
}

Thomas Bonnafont, Rémi Douvenot, Alexandre Chabory

\section{To cite this version:}

Thomas Bonnafont, Rémi Douvenot, Alexandre Chabory. Split-step wavelet with local operators for the 3D long-range propagation. EuCAP 2021 15th European Conference on Antennas and Propagation, Mar 2021, Dusseldorf, Germany. pp.1-5 /ISBN:978-1-7281-8845-4, 10.23919/EuCAP51087.2021.9411173 . hal-03214298

\section{HAL Id: hal-03214298 \\ https://hal-enac.archives-ouvertes.fr/hal-03214298}

Submitted on 11 Oct 2021

HAL is a multi-disciplinary open access archive for the deposit and dissemination of scientific research documents, whether they are published or not. The documents may come from teaching and research institutions in France or abroad, or from public or private research centers.
L'archive ouverte pluridisciplinaire HAL, est destinée au dépôt et à la diffusion de documents scientifiques de niveau recherche, publiés ou non, émanant des établissements d'enseignement et de recherche français ou étrangers, des laboratoires publics ou privés. 


\title{
Split-step wavelet with local operators for the 3D long-range propagation
}

\author{
Thomas BONNAFONT*, Remi DOUVENOT*, Alexandre CHABORY*, \\ *ENAC, Université de Toulouse, Toulouse, FRANCE, thomas.bonnafont@enac.fr
}

\begin{abstract}
Modeling the long-range propagation in the low troposphere is a topic of major concern for many systems such as radar. In 3D, split-step Fourier methods are limited by both computation time and memory size. Therefore, the $\mathbf{N}$ times 2D split-step Fourier method is usually used to model the propagation even if 3D effects are not taken into account. To overcome this, we propose a 3D wavelet-based split-step method that is memory and time efficient. This method is based on the use of the fast wavelet transform, which is of low complexity and allows efficient sparse representations. From numerical experiments, we show that the 3D proposed split-step wavelet method is efficient in terms of precision, time, and memory.
\end{abstract}

Index Terms-split-step, wavelets, 3D long-range propagation, parabolic wave equation.

\section{INTRODUCTION}

The 3D long-range propagation of electromagnetic waves is a topic of major interest in many applications in surveillance, communication, and navigation. In this context, models based on the parabolic wave equation (PWE) [1] are widely used. One efficient method to solve the PWE is split-step Fourier (SSF).

By going back and forth from the spectral to the spatial domain, this method iteratively computes the propagation of the field. The propagation is split into two terms. One term of propagation in a homogenous slice of atmosphere in the spectral domain. Another term to model the refraction with a phase screen in the spatial domain. In 2D this method can accurately model the propagation also taking into account ground composition, with the discrete mixed Fourier transform (DMFT) [2], and relief [3], [4].

In $3 \mathrm{D}, N \times 2 \mathrm{D} \mathrm{SSF}$ methods are usually used. They consist of scanning the domain with a 2D method. Nevertheless, the 3D lateral effects are neglected. Therefore, extensions of SSF to 3D have been developed [5]. Recently, its discrete counterpart (DSSF) in cylindrical coordinates [6] has been developed to avoid spurious solutions. These methods allow to model the $3 \mathrm{D}$ propagation while taking into account $3 \mathrm{D}$ effects. However, they are not memory and time efficient, which prevent their operational use.

In 2D, a wavelet-based split-step method (SSW) has been introduced [7]. Based on the fast wavelet transform (FWT) [8] and the sparse representation allowed by wavelets, this method is fast and memory efficient.

In this article, we propose a generalization of this method to $3 \mathrm{D}$ in order to obtain a usable method for 3D longrange propagation. The main objective is to obtain an efficient method in terms of memory occupation and computation time. In this work, the 3D scalar problem without taking into account relief is studied. Yet, ground composition and refraction are considered.

In Section II, the problem configuration is defined. In Section III, a brief introduction to the 2D multilevel wavelet decomposition on a separable basis is proposed. In Section $\mathrm{IV}$, the 2D wavelet transform is used in the 3D split-step wavelet method. First, an overview of the method is given. Then we focus on the propagation part of the method. In Section V, numerical tests are proposed to validate the method and highlight its advantages in terms of memory occupation and computation time. Finally, Section VI concludes the paper

\section{CONFIGURATION}

In this article, an $\exp (j \omega t)$ dependence is assumed, with $\omega$ the angular frequency. We aim at modeling the 3D forward propagation of the reduced field $u$ in Cartesian coordinates over the ground. The source is located at $x_{s} \leq 0$ and the field is known at $x=0$.

The domain is of finite size $x \in\left[0, x_{\max }\right], y \in\left[0, y_{\max }\right]$ and $z \in\left[0, z_{\max }\right]$. First, a discretization along $y$ and $z$ axis is applied

$$
\begin{aligned}
& y_{p_{y}}=p_{y} \Delta y, p_{y} \in\left[0, N_{y}-1\right] \\
& z_{p_{z}}=p_{z} \Delta z, p_{z} \in\left[0, N_{z}-1\right]
\end{aligned}
$$

with $N_{y}=y_{\max } / \Delta y, N_{z}=z_{\max } / \Delta z$ the number of points in each direction. The discretized reduced field along $y$ and $z$ is denoted by $u_{x}\left[p_{y}, p_{z}\right]$. A discretization along $x$ is also applied with a step $\Delta x$ and $N_{x}=x_{\max } / \Delta x$.

\section{BRIEF INTRODUCTION TO WAVELETS}

Wavelets are widely used in image processing, e.g., JPEG2000 format. In this paper, the 2D multilevel wavelet transform in a separable basis [8] is used to decompose the reduced field $u_{x}\left[p_{y}, p_{z}\right]$. A basis of $3 L+1$ wavelets is used, with $L$ the maximum level. A wavelet is a short length oscillating function of zero-mean. in $2 \mathrm{D}$, three different wavelets are used to describe the horizontal, vertical, and diagonal variations of the signal. These latter are dilated on $L$ levels to take into account slower variations of the signal. A wavelet is denoted by $\psi_{l}^{o}$, with $l$ its level and $o$ its orientation (horizontal, vertical, or diagonal). A last function, the scaling function $\phi$, of non-zero mean is added to take into account the 
slowest variations of the signal. These functions are translated $\left(t_{y}, t_{z}\right)$ to obtain an orthonormal basis.

On this basis, using the FWT [8], the field is decomposed on the wavelet atoms on $L$ levels. This transform is of complexity $O\left(N_{y} N_{z}\right)$, lower than the FFT. Besides, the wavelet coefficients are fastly decreasing to 0 allowing an efficient sparse representation using compression.

\section{SPLiT-STEP WAVELET}

In this section, the 3D split-step wavelet method is introduced. For conciseness, since the scaling function $\phi$ and a $L$-level wavelet are computed likewise, the scaling function is omitted here.

\section{A. Overview of the method}

In this section, an overview of the split-step wavelet (SSW) method is given. This latter is an iterative method that computes the field further from the source by going back and forth in the wavelet and spatial domains. A step of SSW from $x$ to $x+\Delta x$ is performed as follows:

1) The reduced field $u_{x}$ is decomposed in the wavelet domain with a FWT (operator $W$ ). Compression with threshold $V_{s}$ is applied (operator $C$ ). The sparse matrix $U_{x}$ of size $N_{y} \times N_{z}$ containing the wavelet coefficients is obtained

$$
U_{x}=C W u_{x}
$$

2) Propagation in the wavelet domain is achieved using operator $P$

$$
U_{x+\Delta x}=P U_{x}
$$

The propagator corresponds to a wavelet-to-wavelet scattering matrix. This operation is comprehensively introduced through Section IV-B to IV-C. This is based on the use of a set of local propagators to propagate each non-zero coefficient of $U_{x}$.

3) The free-space propagated field $u_{x+\Delta x}^{\mathrm{fs}}$ is obtained by applying an inverse FWT (operator $W^{-1}$ )

$$
u_{x+\Delta x}^{\mathrm{fs}}=W^{-1} U_{x+\Delta x} .
$$

Thus, $u_{x+\Delta x}^{\mathrm{fs}}$ is expressed in the space domain.

4) The refraction is taken into account using the phase screen method [2] (operator $R$ ) to obtain the field at $x+\Delta x$

$$
u_{x+\Delta x}=R u_{x+\Delta x}^{\mathrm{fs}} .
$$

A PEC ground is taken into account with few additional points using the local image method [9]. In the case of an c ground, the local image method with the DMFT change of variable is applied.e

Finally, the domain is limited at each side in $y$-direction and at the top in $z$-direction. An apodization with the Hanning window is applied to model absorbing conditions toward infinity.

\section{B. Computing the local propagators}

In this section, we introduce how to efficiently compute the set of local propagators. This set is constructed following and adapting the 2D strategy [7], which stores the essential wavelet-to-wavelet propagations.

The computation of this set is performed in 3 steps as follows:

1) For each level $l$ and orientation $o$ the wavelet $\chi_{l, 0}^{o}\left[p_{y}, p_{z}\right]$ is propagated of $\Delta x$ using the 3D DSSF [6]. Thus, $\psi_{l, \Delta x}^{o}\left[p_{y}, p_{z}\right]$ is obtained. Thus, a total of $3 L+1$ DSSF is needed.

2) Since, as in 1D [9], the space grids of the wavelets are dilated by 2 at each level in each direction, translations of $\left(p_{t_{y}}^{l}, p_{t, z}^{l}\right) \in\left[0,2^{L-l}\left[\times\left[0,2^{L-l}[\right.\right.\right.$ in $y$ and $z$ directions are required. The translated propagated wavelet $\psi_{l, \Delta x}^{o}\left[p_{y}+p_{t_{y}}^{l}, p_{z}+p_{t_{z}}^{l}\right]$ is thus obtained. This leads to $2^{2(L-l)}$ translations for each level.

3) The propagated wavelet field $\psi_{l, \Delta x}^{o}\left[p_{y}+p_{t_{y}}^{l}, p_{z}+p_{t_{z}}^{l}\right]$ is decomposed with a 2D FWT and compressed (threshold $V_{p}$ ) to obtain propagator $P_{l, o, p_{t y}^{l}, p_{t_{z}}^{l}}$. Hence a total of only $1+3 \sum_{l=1}^{L} 2^{2(L-l)}$ sparse wavelet-to-wavelet propagations are stored in $P$. This corresponds to the minimal number of necessary propagations.

The support of the wavelet is smaller than the overall domain and theoretically known [8]. Therefore computing the propagation of the wavelet on its own support induces a drastic reduction of the computation time [7]. Besides, if $\Delta y=\Delta z$ the vertical wavelet corresponds to a rotation of $90^{\circ}$ of the horizontal wavelet. Using this rotation invariance, the propagation of the vertical wavelet can be computed as the rotation of the propagated horizontal wavelet. This yields to only $2 L+1$ necessary DSSF propagations instead of $3 L+1$, reducing further the computation time.

\section{Propagation using the local propagators}

In this part, the method to compute the propagation using the set of local propagators $P$ is introduced.

First, the non-zero wavelet coefficients of $U_{x}$, denoted by $\alpha_{l}^{o}\left[p_{y}, p_{z}\right]$, are obtained by applying a FWT and a compression to the field $u_{x}$. Second, for each of this coefficient, we select the associated local propagator $P_{l, o, p_{t y}, p_{t_{z}}^{l}}$ with

$$
\begin{array}{ll}
p_{t_{y}}^{l}=p_{y} & \bmod 2^{L-l}, \\
p_{t_{z}}^{l}=p_{z} & \bmod 2^{L-l},
\end{array}
$$

$l$ and $o$ the level and orientation of $\alpha$, respectively. Third, the free-space elementary propagation associated with this coefficient corresponds to $\alpha_{l}^{o}\left[p_{y}, p_{z}\right] P_{l, o, p_{t_{y}}^{l}, p_{t_{z}}^{l}}$. Finally, these local propagations are translated to the position of the coefficient and summed up to obtain the propagated wavelet coefficients

$$
U_{x+\Delta x}=\sum_{l, o, p_{y}, p_{z}}\left(\alpha_{l}^{o}\left[p_{y}, p_{z}\right] P_{l, o, p_{t_{y}}^{l}, p_{t_{z}}^{l}}\right)\left[\cdot+p_{y}, \cdot+p_{z}\right] \text {. }
$$

With these steps, the propagated wavelet coefficients $U_{x+\Delta x}$ are obtained. An inverse FWT yields the free-space propagated field. 


\section{NUMERICAL EXPERIMENTS}

The objective of the numerical experiments is to test the method and compare it to DSSF in terms of computation time and memory occupation.

\section{A. Free-space propagation}

In this test, we aim at validating the method with a freespace propagation. To do so, the propagation in free space of the field radiated by a CSP at $300 \mathrm{MHz}$ is studied. The domain is of size $x_{\max }=1500 \mathrm{~m}$ and $y_{\max }=z_{\max }=1024 \mathrm{~m}$. The steps are set to $\Delta x=50 \mathrm{~m}$ and $\Delta y=\Delta z=0.5 \mathrm{~m}$. The source is placed at $x_{s}=-50 \mathrm{~m}$ and $y_{s}=z_{s}=512 \mathrm{~m}$. The waist size is $W_{0}=5 \mathrm{~m}$.

For the wavelet parameters, we choose the symlet family with 6 vanishing moments and a maximum level $L=3$. The thresholds are set to $V_{s}=V_{p}=5.3 \times 10^{-4}$.

The propagation is computed both with SSW and SSF. Firstly, in Figure 1 (a) the normalized field obtained with SSW in the $y O z$-plane is plotted. Secondly, Figure 1 (b) shows the normalized field obtained with SSW in the $x O z$-plane. Finally, in Figure 1 (c) the root mean square (RMS) difference in $\mathrm{dB}$ between SSW and DSSF is presented.

The final normalized difference between both methods is below $-45 \mathrm{~dB}$. This error and the RMS difference is due to the two compressions (of thresholds $V_{s}$ and $V_{p}$ ) that induce an accumulation of error throughout the propagation. Nevertheless, differences are acceptable and can be managed using the thresholds. Therefore, the method is working correctly in this case.

In terms of computation time and memory occupation, results are summed up in Table I. In this latter, propagator time or memory correspond to the set of local propagators for SSW and to storing the propagation matrix for DSSF. The propagation time and memory correspond to the time to compute the propagation on the overall domain and to the memory needed to store the propagated field or coefficients, respectively.

TABLE I: Computation time and memory occupation needed by SSW and DSSF.

\begin{tabular}{|c|c|c|}
\hline method & DSSF & SSW \\
\hline propagator time (s) & 138 & 8.3 \\
\hline propagation time (s) & 343 & 330 \\
\hline propagator memory (MB) & 604 & 10.2 \\
\hline propagation memory (MB) & 604 & 0.05 to 2.1 \\
\hline
\end{tabular}

We can conclude that in this case, SSW is comparable in terms of computation time to SSF. Nevertheless, the memory occupation is better with SSW both to store the propagator or the propagated wavelet coefficients. This shows the advantage of SSW over DSSF.

\section{B. Bidirectional-duct scenario}

Now a more complex scenario with 3D lateral effects along $y$ and $z$ is studied. The propagation of the field radiated by a CSP at $300 \mathrm{MHz}$ with a bidirectional-duct is modeled. With

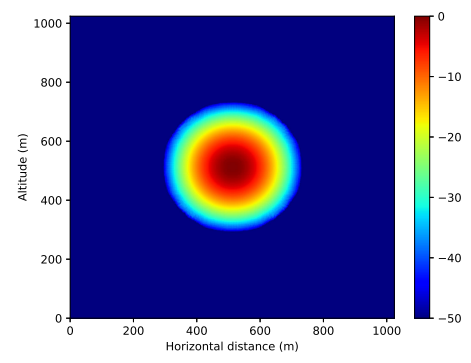

(a) Final normalized field $(\mathrm{dB})$ obtained with ISSW in $y O z$-plane

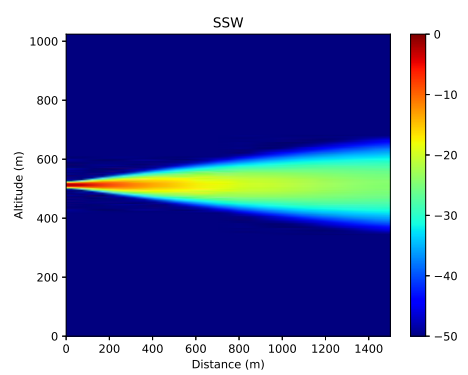

(b) Final normalized field $(\mathrm{dB})$ obtained with ISSW in $x O z$-plane

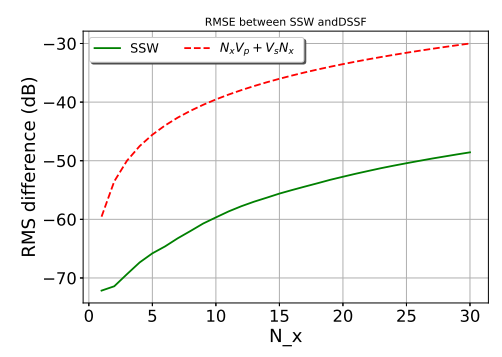

(c) RMS difference evolution (dB).

Fig. 1: Propagation of the field radiated by a CSP in free space with.

the 3D method, we expect to obtain the same results in both directions, whereas a $N \times 2 \mathrm{D}$ method could only be accurate in one direction.

The configuration parameters are as follows. The propagation is computed over $10 \mathrm{~km}$ with steps of $200 \mathrm{~m}$ in $x$ direction. The domain is of size $2048 \times 2048 \mathrm{~m}$ in $y$ and $z$ directions with $\Delta y=\Delta z=1 \mathrm{~m}$. The source is a complex source point placed at $x_{s}=-50 \mathrm{~m}$ with a waist size of $W_{0}=10 \mathrm{~m}$ and at $y_{s}=z_{s}=1024 \mathrm{~m}$.

The wavelet parameters remain the same, except for the thresholds $V_{s}=V_{p}=10^{-3}$.

The bidirectional-duct is modeled with tri-linear models with the same parameters in both $y$ and $z$ directions. The parameters for the two ducts are: $M_{0}=330$ M-units, $y_{b}=$ $z_{b}=950 \mathrm{~m}, y_{t}=z_{t}=100 \mathrm{~m}, c_{0}=0.118 \mathrm{M}$-units $/ \mathrm{m}$ and $c_{2}=-1 \mathrm{M}$-units $/ \mathrm{m}$. The corresponding modified refractive 
index $M$ is plotted in Figure 2. This corresponds to the phase screen applied at each step.

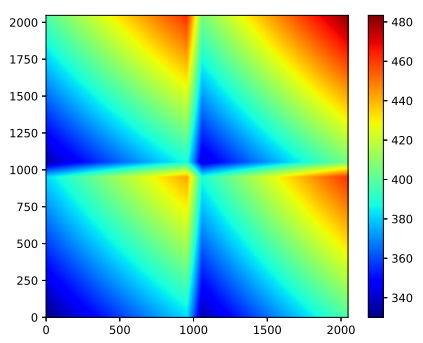

Fig. 2: M-index (M-units $/ \mathrm{m})$ on the $y O z$-plane.

As previously, the propagation is computed both with SSW and SSF. Firstly, in Figure 3 (a) the final normalized field obtained with SSW in the $y O z$-plane is plotted. Secondly, Figure 3 (b) shows the final normalized difference between SSW and DSSF in the $y O z$-plane. Finally, in Figure 3 (c) horizontal and vertical cuts of the field at $N_{y} / 2$ and $N_{z} / 2$ are presented.

We can observe that the 3D effects due to the refraction are accurately simulated. Indeed, in both directions, the results match within an acceptable error. Besides the normalized error with DSSF is below $-30 \mathrm{~dB}$, which is negligible. Thus, SSW has been successfully tested in a $3 \mathrm{D}$ long-range propagation scenario with complex atmospheric effects.

In terms of computation time and memory occupation, the results are summed up in Table II.

TABLE II: Computational cost of 1SSW and DSSF in terms of computation time and memory occupation.

\begin{tabular}{|c|c|c|}
\hline method & DSSF & 1SSW \\
\hline propagator time (s) & 133 & 6.5 \\
\hline propagation time (min) & 9.2 & 10 \\
\hline propagator memory (MB) & 604 & 5.1 \\
\hline propagation memory (MB) & 604 & 0.03 to 3.4 \\
\hline
\end{tabular}

In this case, the computation time is slightly better with DSSF. This is due to the propagation part. In DSSF this part uses the optimized Python matrix-vector product, whereas SSW is a Python laboratory code with loops. Nevertheless, the memory occupation is better with SSW by a factor of 100 , showing the advantage of SSW for 3D long-range propagation.

\section{CONCLUSION}

In this paper, a wavelet-based split-step method for modeling the 3D long-range propagation has been proposed.

This method iteratively computes the field by going back and forth in the wavelet and spatial domains. The method is based on the propagation of the wavelet coefficients of the field using a set of local propagators. Using the advantages of the wavelet transform, this method yields a fast and memoryefficient method. Numerical experiments have been performed to validate the method. First, propagation in a homogeneous

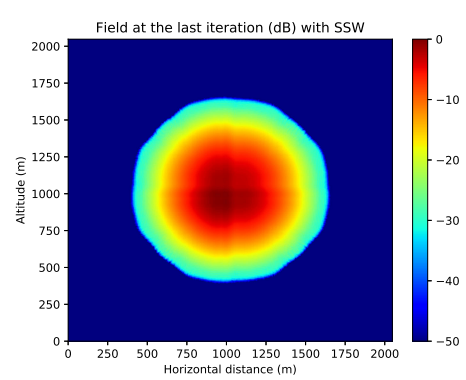

(a) Final normalized field $(\mathrm{dB})$ obtained with ISSW in $y O z$-plane.

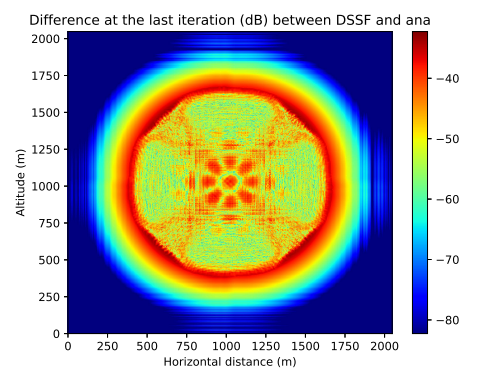

(b) Final normalized difference $(\mathrm{dB})$ between ISSW and DSSF in $y O z$-plane

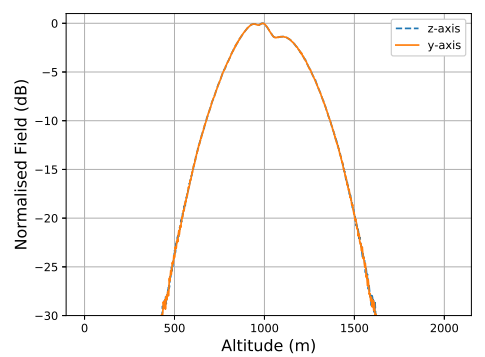

(c) Vertical/horizontal cuts of the final normalized field $(\mathrm{dB})$ obtained with 1SSW.

Fig. 3: Propagation of the field radiated by a CSP in a bidirectional-duct scenario.

atmosphere has shown that the method is accurate and efficient. Second, a long-range propagation scenario with a bidirectional duct has shown that the method is as accurate as DSSF. Besides SSW is better in terms of memory occupation than DSSF. Nevertheless, the computation time is of the same order and should be improved.

Future works include implementing the wavelet propagation in a compiled language for future comparison with DSSF. Also, the problem of taking into account the relief in $3 \mathrm{D}$ should be considered.

\section{ACKNOWLEDGMENT}

The authors would like to thank the French Defense Agency (Direction Général de l'Armement, DGA) and the French Civil Aviation University (École Nationale de l'Aviation Civile, ENAC) for the funding. 


\section{REFERENCES}

[1] M. Levy, Parabolic Equation Methods for Electromagnetic Wave Propagation, IET, 2000

[2] D. Dockery and J. R. Kuttler, “ An improved impedance-boundary algorithm for Fourier split-step solutions of the parabolic wave equation,' IEEE Transactions on Antennas and Propagation, 44, pp. 1592-1599, 1996.

[3] A. E. Barrios, "A terrain parabolic equation model for propagation in the troposphere," IEEE Transactions on Antennas and Propagation, 42(1), pp 90-98, 1994.

[4] R. Janaswamy, "A curvilinear coordinate-based split-step parabolic equation method for propagation predictions over terrain," IEEE Transactions on Antennas and Propagation, 46(7), pp 1089-1097, 1998.

[5] R. Janaswamy, "Path loss predictions in the presence of buildings on flat terrain: A 3-D vector parabolic equation approach," IEEE Transactions on Antennas and Propagation, 51(8), pp 1716-1728, 2003.

[6] H. Zhou, A. Chabory, and R. Douvenot. "A 3-D split-step Fourier algorithm based on a discrete spectral representation of the propagation equation," IEEE Transactions on Antennas and Propagation, 65(4), pp 1988-1995, 2017.

[7] T. Bonnafont, R. Douvenot, and A. Chabory,“ A speed-up of split-step wavelet for the computation of long-range propagation," 2020 14th European Conference on Antennas and Propagation (EUCAP), IEEE, 2020.

[8] S. Mallat, A Wavelet Tour of Signal Processing, Academic press, 1999.

[9] H. Zhou, R. Douvenot, and A. Chabory. "Modeling the long-range wave propagation by a split-step wavelet method," Journal of Computational Physics, 402:109042, 2020 